Radiologe $2016 \cdot 56: 959$

DOI 10.1007/s00117-016-0178-7

() Springer-Verlag Berlin Heidelberg 2016

CrossMark

\title{
W. Reith
}

Klinik für Diagnostische und Interventionelle Neuroradiologie, Universitätsklinikum des Saarlandes, Homburg/Saar, Deutschland

\section{Hintere Schädelgrube}

Liebe Leserinnen und Leser,

in der hinteren Schädelgrube sind auf engem Raum wichtige Strukturen zusammengefasst. Hier gibt es zahlreiche anatomische Varianten und Fehlbildungen, wie z. B. die Chiari-Malformation, sowie zerebelläre Hypo- und Dysplasien. Neben diesen zahlreichen Varianten gibt es auch wichtige und häufig vorkommende Tumoren der hinteren Schädelgrube, wie z.B. das Akustikusneurinom oder Meningeome.

Da wenig Platz in der hinteren Schädelgrube existiert, führen bereits kleine Tumoren mit geringen Raumforderungen zu einer erheblichen klinischen Symptomatik. Daneben gibt es auch zahlreiche Gefäßfehlbildungen der hinteren Schädelgrube wie Aneurysmen oder arteriovenöse(AV)-Malformationen.

In der CT-Ära war die hintere Schädelgrube aufgrund der Artefakte immer relativ schlecht zu beurteilen. Erst durch die MRT-Technik kann die hintere Schädelgrube mit großer Genauigkeit abgebildet werden.

Daneben gibt es auch klinisch relevante neurodegenerative Erkrankungen mit Beteiligung des Kleinhirns. Diese führen z. T. zu charakteristischen Symptomen, wiez. B. Ataxie, Augenbewegungsstörungen, Dysarthrie und Dysmetrie.

Wichtig für den in der Befundung tätigen Radiologen ist, dass er die anatomischen Strukturen und Details der hinteren Schädelgrube kennt und auch die zahlreichen dort vorkommenden
Varianten von unter Umständen pathologischen behandlungsbedürftigen Erkrankungen abgrenzen kann.

Ihr

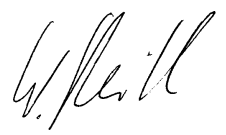

Prof. Dr. Wolfgang Reith

\section{Korrespondenzadresse}

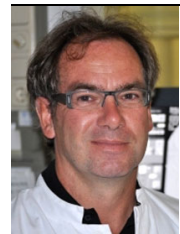

Prof. Dr. W. Reith

Klinik für Diagnostische und Interventionelle Neuroradiologie, Universitätsklinikum des Saarlandes Kirrberger Straße 1, 66424 Homburg/Saar, Deutschland Wolfgang.Reith@ uniklinikum-saarland.de

Interessenkonflikt. W. Reith gibt an, dass kein Interessenkonflikt besteht. 